\title{
Correlative large volume imaging across scales
}

\author{
Rasmus R. Schröder ${ }^{1,3}$, Holger Blank ${ }^{2}$, Andreas Schertel ${ }^{2}$, Marlene Thaler ${ }^{2}$, Alexander Orchowski ${ }^{2}$, \\ and Irene Wacker ${ }^{3}$ \\ ${ }^{1}$ Cryo EM, CellNetworks, BioQuant, Universitätsklinikum Heidelberg, Heidelberg, Germany \\ ${ }^{2}$ Carl Zeiss Microscopy GmbH, Oberkochen, Germany \\ ${ }^{3}$ Cryo EM, Centre for Advanced Materials, Universität Heidelberg, Heidelberg, Germany
}

For correlative imaging of large volumes integrated workflows allowing shuttling of samples between different imaging modalities would be ideal. X-ray microscopy (XRM) is one modality, which in principle can bridge the gap between macroscopic and microscopic world. Here we have started to assess how XRM, light microscopy (LM) and electron microscopy (EM) might be integrated to gain comprehensive information about biological samples extended in 3D.

In CLEM, correlated LM and EM, fluorescence LM is commonly used to define a certain functional state, which is then put into structural context using EM. That this is also possible for XRM has been shown for single cells grown on TEM grids which were plunge frozen and analyzed by cryo-XRM [1]. For tissue samples such an approach is not possible because samples thicker than 200 micrometers cannot be vitrified. In that case a different workflow is required, starting with a chemical fixation. In LM and XRM samples may be then imaged directly in aqueous medium, XRM being able to yield voxel sizes down to $350 \mathrm{~nm}$. However, if ultrastructural resolution in the range of few nanometers is required XRM needs to be complemented by EM. There are several options to achieve that for large volumes [2] based on SEM imaging such as serial blockface or focussed ion beam scanning electron microscopy (SBFSEM or FIBSEM) or array tomography (AT). SBFSEM is optimal for connectomics where whole brains need to be imaged at high resolution. In other areas of cell and developmental biology the biggest part of the sample surface being imaged may not be interesting for the question being addressed, only a minute part of it may contain the target structure. So the question of how to identify this target is of eminent importance. Here we employ XRM to identify a rare event such as the formation of an immunological synapse (IMS) or a rare structure such as the neuromuscular junction (NMJ) within a large volume. We are using the new solution ZEISS Atlas 5 (Carl Zeiss Microscopy GmbH) which offers a sample centric correlative environment fusing all available $2 \mathrm{D}$ and $3 \mathrm{D}$ data of the sample from various modalities. It contains modules for automated SEM large area imaging and targeted crossbeam (XB) nanotomography. Based on a large volume XRM dataset it thus permits an efficient approach for nanometer scale analysis of identified buried features of interest using Crossbeam technology.

Figure 1 illustrates typical workflows for large volume samples such as muscle tissue or cell pellets. Important is the definition of a reference framework, which is inherent to the samples of interest. This "sample coordinate system" needs to be mapped seamlessly from one imaging technique to the next, which then allows for a fast and accurate navigation of the sample in 3D.

[1] Hagen et al., J Struct Biol 177 (2012) 193-201; Chicon et al., J Struct Biol 177 (2012) 202-211.

[2] I Wacker, RR Schröder, J Microscopy 252 (2013) 93-99.

[3] The authors acknowledge C Bartels and L Veith for technical support, C Grabher for samples, BMBF for NanoCombine grant FKZ 13N11401 and MorphiQuant grant FKZ 13GW0044. 


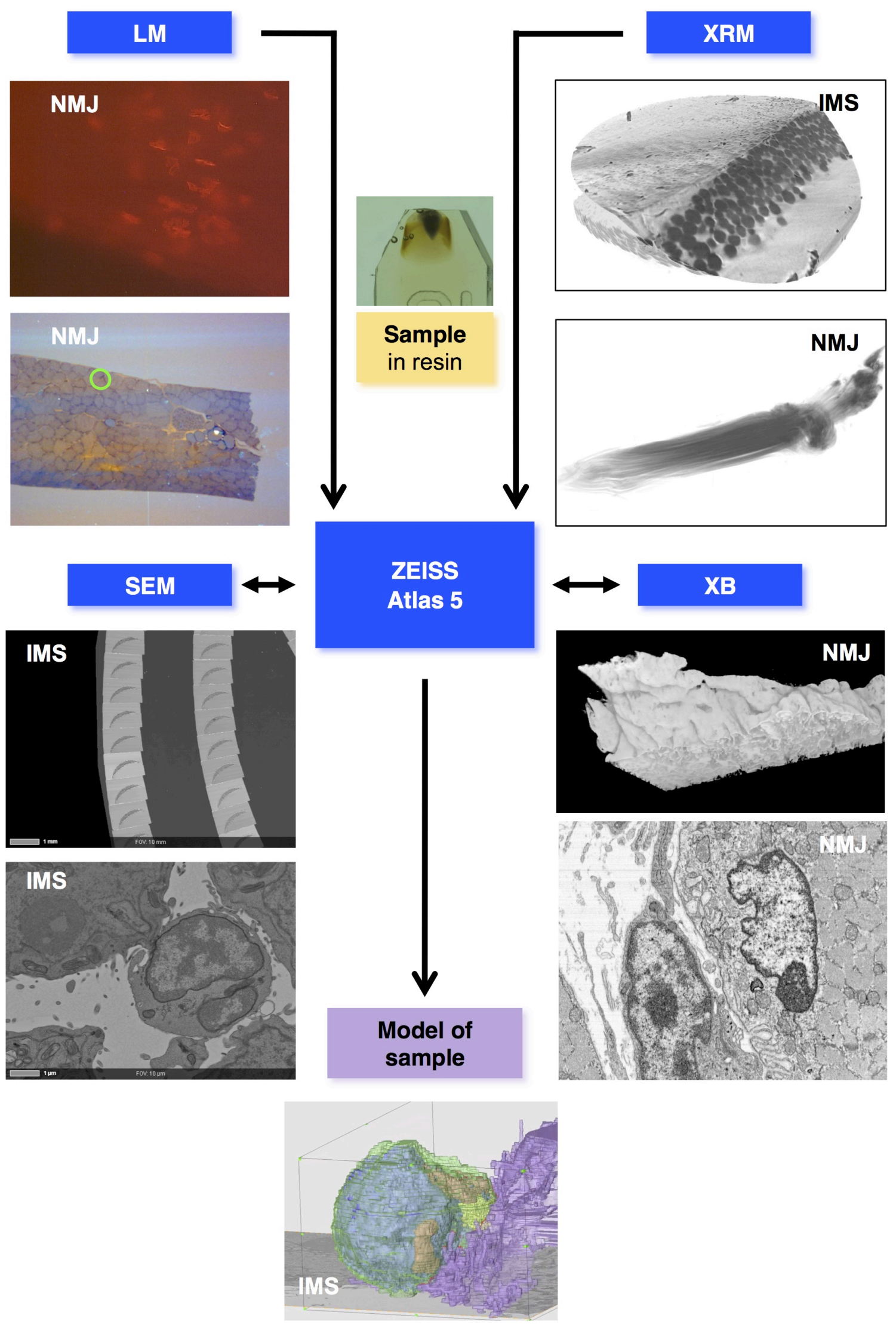

